\title{
Rank-Maximal Matchings - Structure and Algorithms
}

\author{
Pratik Ghosal ${ }^{1}$, Meghana Nasre ${ }^{2}$, and Prajakta Nimbhorkar ${ }^{\star 3}$ \\ 1 University of Wrocław, Poland (pratikghosal20082@gmail.com) \\ 2 Indian Institute of Technology Madras (meghana@cse.iitm.ac.in) \\ 3 Chennai Mathematical Institute (prajakta@cmi.ac.in)
}

\begin{abstract}
Let $G=(\mathcal{A} \cup \mathcal{P}, E)$ be a bipartite graph where $\mathcal{A}$ denotes a set of agents, $\mathcal{P}$ denotes a set of posts and ranks on the edges denote preferences of the agents over posts. A matching $M$ in $G$ is rank-maximal if it matches the maximum number of applicants to their top-rank post, subject to this, the maximum number of applicants to their second rank post and so on.

In this paper, we develop a switching graph characterization of rankmaximal matchings, which is a useful tool that encodes all rank-maximal matchings in an instance. The characterization leads to simple and efficient algorithms for several interesting problems. In particular, we give an efficient algorithm to compute the set of rank-maximal pairs in an instance. We show that the problem of counting the number of rankmaximal matchings is \#P-Complete and also give an FPRAS for the problem. Finally, we consider the problem of deciding whether a rankmaximal matching is popular among all the rank-maximal matchings in a given instance, and give an efficient algorithm for the problem.
\end{abstract}

\section{Introduction}

We consider the problem of matching applicants to posts where applicants have preferences over posts. This problem is motivated by several important real-world applications like allocation of graduates to training positions [4] and families to government housing [15. The input to the problem is a bipartite graph $G=$ $(\mathcal{A} \cup \mathcal{P}, E)$, where $\mathcal{A}$ is a set of applicants, $\mathcal{P}$ is a set of posts, and the set $E$ can be partitioned as $E=E_{1} \cup \ldots \cup E_{r}$, where $E_{i}$ contains the edges of rank $i$. An edge $(a, p) \in E_{i}$ if $p$ is an $i$ th choice of $a$. An applicant $a$ prefers a post $p$ to $p^{\prime}$ if, for some $i<j,(a, p) \in E_{i}$ and $\left(a, p^{\prime}\right) \in E_{j}$. Applicant $a$ is indifferent between $p$ and $p^{\prime}$ if $i=j$. This ranking of posts by an applicant is called the preference list of the applicant. When applicants can be indifferent between posts, preference lists are said to contain ties, else preference lists are strict.

The problem of matching under one-sided preferences has received lot of attention and there exist several notions of optimality like pareto-optimality [1],

\footnotetext{
* Part of the work has been done while the author was on a sabbatical to the Institute of Mathematics of the Czech Academy of Sciences, Prague.
} 
rank-maximality [6], popularity [2], and fairness. We focus on the well-studied notion of rank-maximal matchings which are guaranteed to exist in any instance. Rank-maximality was first studied under the name of greedy matchings by Irving [5, who also gave an algorithm for computing such matchings in case of strict lists. A rank-maximal matching matches maximum number of applicants to their rank 1 posts, subject to that, maximum number of applicants to their rank 2 posts and so on. Irving et al. [6] gave an $O(\min (n+r, r \sqrt{n}) m)$ time algorithm to compute a rank-maximal matching. This algorithm $[\underline{6}$ not only works for strict case, but also for tied case. Here $n=|\mathcal{A}|+|\mathcal{P}|, m=|E|$, and $r$ denotes the maximal rank in the instance. The weighted and capacitated versions of this problem have been studied in [10] and [13] respectively.

In this paper, we study the structure of the rank-maximal matchings using the notion of a switching graph. This notion was introduced in the context of popularity which is an alternative criterion of optimality in the one-sided preferences model. See [2] for a definition of popular matchings. McDermid and Irving [1] studied the switching graph of popular matchings for strict instances, and Nasre [12] extended it to the case of ties. This characterization has turned out to be useful for several problems like counting the number of popular matchings in strict instances, computing an optimal popular matching, developing an optimal manipulation strategy for an agent etc.

It is natural to extend the switching graph characterization to analyze rankmaximal matchings. Besides being interesting in its own right, it turns out to be useful in answering several natural questions. For instance, given instance $G=(\mathcal{A} \cup \mathcal{P}, E)$, is there a rank-maximal matching in $G$ which matches an applicant $a$ to a particular post $p$ ? Is a rank-maximal matching preferred by a majority of applicants over other rank-maximal matchings in the instance? We show the following new results in this paper:

- A switching graph characterization of the rank-maximal matchings problem, and its properties, using which, we answer the questions mentioned above.

- An efficient algorithm for computing the set of rank-maximal pairs. An edge $(a, p) \in E$ is a rank-maximal pair if there exists a rank-maximal matching in $G$ that matches $a$ to $p$.

- We show that the problem of counting the number of rank-maximal matchings is \#P-complete even for strict preference lists. We then give an FPRAS for the problem by reducing it to the problem of counting the number of perfect matchings in a bipartite graph.

- In order to choose one among possibly several rank-maximal matchings in a given instance $G$, we consider the question of finding a rank-maximal matching that is popular among all the rank-maximal matchings in $G$. We call such a matching a popular rank-maximal matching. We show that, given a rank-maximal matching, it can be efficiently checked whether it is a popular rank-maximal matching. If not, we output a rank-maximal matching which is more popular than the given one.

We remark that the switching graph is a weighted directed graph constructed with respect to a particular matching. In case of popular matchings, it is known 
from [2] that, there are at most two distinct ranked posts in an applicant's preference list, to which he can be matched in any popular matching. This results in a switching graph with edge-weights $\{+1,-1,0\}$. In case of rank-maximal matchings, the situation becomes more interesting since an applicant can be matched to one among several distinct ranked posts, and the edge-weights in the switching graph could be arbitrary. Surprisingly, the characterization still turns out to be similar to that of popular matchings, although the proofs are significantly different. We expect that the switching graph will find several applications apart from those shown in this paper.

\section{Preliminaries}

A matching $M$ of $G$ is a subset of edges, no two of which share an end-point. For a matched vertex $u$, we denote by $M(u)$ its partner in $M$.

Properties of maximum matchings in bipartite graphs: Let $G=(\mathcal{A} \cup \mathcal{P}, E)$ be a bipartite graph and let $M$ be a maximum matching in $G$. The matching $M$ defines a partition of the vertex set $\mathcal{A} \cup \mathcal{P}$ into three disjoint sets, defined below:

Definition 1 (Even, odd, unreachable vertices). A vertex $v \in \mathcal{A} \cup \mathcal{P}$ is even (resp. odd) if there is an even (resp. odd) length alternating path with respect to $M$ from an unmatched vertex to $v$. A vertex $v$ is unreachable if there is no alternating path from an unmatched vertex to $v$.

The following lemma is well-known in matching theory; see [14] or [6] for a proof.

Lemma 1 ([14]). Let $\mathcal{E}, \mathcal{O}$, and $\mathcal{U}$ be the sets of even, odd, and unreachable vertices defined by a maximum matching $M$ in $G$. Then,

(a) $\mathcal{E}, \mathcal{O}$, and $\mathcal{U}$ are disjoint, and are the same for all the maximum matchings in $G$.

(b) In any maximum matching of $G$, every vertex in $\mathcal{O}$ is matched with a vertex in $\mathcal{E}$, and every vertex in $\mathcal{U}$ is matched with another vertex in $\mathcal{U}$. The size of a maximum matching is $|\mathcal{O}|+|\mathcal{U}| / 2$.

(c) No maximum matching of $G$ contains an edge with one end-point in $\mathcal{O}$ and the other in $\mathcal{O} \cup \mathcal{U}$. Also, $G$ contains no edge with one end-point in $\mathcal{E}$ and the other in $\mathcal{E} \cup \mathcal{U}$.

Rank-maximal matchings: An instance of the rank-maximal matchings problem consists of a bipartite graph $G=(\mathcal{A} \cup \mathcal{P}, E)$, where $\mathcal{A}$ is a set of applicants, $\mathcal{P}$ is a set of posts, and $E$ can be partitioned as $E_{1} \cup E_{2} \cup \ldots \cup E_{r}$. Here $E_{i}$ denotes the edges of rank $i$, and $r$ denotes the maximum rank any applicant assigns to a post. An edge $(a, p)$ has rank $i$ if $p$ is an $i$ th choice of $a$.

Definition 2 (Signature). The signature of a matching $M$ is defined as an $r$-tuple $\rho(M)=\left(x_{1}, \ldots, x_{r}\right)$ where, for each $1 \leq i \leq r, x_{i}$ is the number of applicants who are matched to their ith rank post in $M$. 
Let $M, M^{\prime}$ be two matchings in $G$, with signatures $\rho(M)=\left(x_{1}, \ldots, x_{r}\right)$ and $\rho\left(M^{\prime}\right)=\left(y_{1}, \ldots, y_{r}\right)$. Define $M \succ M^{\prime}$ if $x_{i}=y_{i}$ for $1 \leq i<k \leq r$ and $x_{k}>y_{k}$.

Definition 3 (Rank-maximal matching). A matching $M$ in $G$ is rankmaximal if $M$ has the maximum signature under the above ordering $\succ$.

Observe that all the rank-maximal matchings in an instance have the same cardinality and the same signature.

Computing Rank-maximal Matchings: Now we recall Irving et al.'s algorithm [6] for computing a rank-maximal matching in a given instance $G=\left(\mathcal{A} \cup \mathcal{P}, E_{1} \cup\right.$ $\left.\ldots \cup E_{r}\right)$. Recall that $E_{i}$ is the set of edges of rank $i$. For the sake of convenience, for each applicant $a$, we add a dummy last-resort post $\ell(a)$ at rank $r+1$ in $a$ 's preference list, and refer to the modified instance as $G$. This ensures that every rank-maximal matching is $\mathcal{A}$-complete i.e. matches all the applicants.

Let $G_{i}=\left(\mathcal{A} \cup \mathcal{P}, E_{1} \cup \ldots \cup E_{i}\right)$. The algorithm starts with $G_{1}^{\prime}=G_{1}$ and any maximum matching $M_{1}$ in $G_{1}^{\prime}$.

For $i=1$ to $r$ do the following and output $M_{r+1}$ :

1. Partition the vertices in $\mathcal{A} \cup \mathcal{P}$ into even, odd, and unreachable as in Definition 1 and call these sets $\mathcal{E}_{i}, \mathcal{O}_{i}, \mathcal{U}_{i}$ respectively.

2. Delete those edges in $E_{j}, j>i$, which are incident on nodes in $\mathcal{O}_{i} \cup \mathcal{U}_{i}$. These are the nodes that are matched by every maximum matching in $G_{i}^{\prime}$.

3. Delete all the edges from $G_{i}^{\prime}$ between a node in $\mathcal{O}_{i}$ and a node in $\mathcal{O}_{i} \cup \mathcal{U}_{i}$. We refer to these edges as $\mathcal{O}_{i} \mathcal{O}_{i}$ and $\mathcal{O}_{i} \mathcal{U}_{i}$ edges respectively. These are the edges which do not belong to any maximum matching in $G_{i}^{\prime}$.

4. Add the edges in $E_{i+1}$ to $G_{i}^{\prime}$ and call the resulting graph $G_{i+1}^{\prime}$.

5. Determine a maximum matching $M_{i+1}$ in $G_{i+1}^{\prime}$ by augmenting $M_{i}$.

The algorithm constructs a graph $G_{r+1}^{\prime}$. We construct a reduced graph $G^{\prime}$ by deleting all the edges from $G_{r+1}^{\prime}$ between a node in $\mathcal{O}_{r+1}$ and a node in $\mathcal{O}_{r+1} \cup \mathcal{U}_{r+1}$. The graph $G^{\prime}$ will be used in subsequent sections.

We note the following invariants of Irving et al.'s algorithm:

(I1) For every $1 \leq i \leq r$, every rank-maximal matching in $G_{i}$ is contained in $G_{i}^{\prime}$.

(I2) The matching $M_{i}$ is rank-maximal in $G_{i}$, and is a maximum matching in $G_{i}^{\prime}$.

(I3) If a rank-maximal matching in $G$ has signature $\left(s_{1}, \ldots, s_{i}, \ldots s_{r}\right)$ then $M_{i}$ has signature $\left(s_{1}, \ldots, s_{i}\right)$.

(I4) The graphs $G_{i}^{\prime}, 1 \leq i \leq r+1$ constructed at the end of iteration $i$ of Irving et al.'s algorithm, and $G^{\prime}$ are independent of the rank-maximal matching computed by the algorithm. This follows from Lemma 1 and invariant $I 2$.

\section{Switching Graph Characterization}

In this section, we describe the switching graph characterization of rank-maximal matchings, and show its application in computing rank-maximal pairs.

Let $M$ be a rank-maximal matching in $G$ and let $G^{\prime}=\left(\mathcal{A} \cup \mathcal{P}, E^{\prime}\right)$ be the reduced graph as described in Section 2 . 
Definition 4 (Switching Graph). The switching graph $G_{M}=\left(V_{M}, E_{M}\right)$ with respect to a rank-maximal matching $M$ is a directed weighted graph with $V_{M}=\mathcal{P}$ and $E_{M}=\left\{\left(p_{i}, p_{j}\right) \mid \exists a \in \mathcal{A},\left(a, p_{i}\right) \in M,\left(a, p_{j}\right) \in E^{\prime}\right\}$. Further, weight of an edge $\left(p_{i}, p_{j}\right)$ is $w\left(p_{i}, p_{j}\right)=\operatorname{rank}\left(a, p_{j}\right)-\operatorname{rank}\left(a, p_{i}\right)$, where $\operatorname{rank}(a, p)$ is the rank of a post $p$ in the preference list of an applicant $a$.

Thus an edge $\left(p_{i}, p_{j}\right) \in E_{M}$ iff there exists an applicant $a$ such that $\left(a, p_{i}\right) \in$ $M$ and $\left(a, p_{j}\right)$ is an edge in the graph $G^{\prime}$. We define the following notation:

1. Sink vertex: A vertex $p$ of $G_{M}$ is called a sink vertex, if $p$ has no outgoing edge in $G_{M}$ and $p \in \mathcal{E}_{1} \cap \mathcal{E}_{2} \cap \ldots \cap \mathcal{E}_{r+1}$. Recall that $\mathcal{E}_{i}$ is the set of vertices which were even in the graph $G_{i}^{\prime}$ constructed in the $i$ th iteration of Irving et al.'s algorithm.

2. Sink and non-sink components of $G_{M}$ : A connected component $\mathcal{X}$ in the underlying undirected graph of $G_{M}$ is called a sink component if $\mathcal{X}$ contains one or more sink vertices, and a non-sink component otherwise.

3. Switching paths and switching cycles: A path $T=\left\langle p_{0}, p_{1} \ldots, p_{k-1}\right\rangle$ in $G_{M}$ is called a switching path if $T$ ends in a sink vertex and $w(T)=0$. Here, $w(T)$ is the sum of the weights of the edges in $T$. A cycle $C=\left\langle p_{0}, \ldots, p_{k-1}, p_{0}\right\rangle$ in $G_{M}$ is called a switching cycle if $w(C)=0$.

4. Switching operation: Let $T=\left\langle p_{0}, p_{1} \ldots, p_{k-1}\right\rangle$ be a switching path in $G_{M}$. Let $\mathcal{A}_{T}=\{a \in \mathcal{A} \mid M(a) \in T\}$. Further, let $M\left(a_{i}\right)=p_{i}$ for $0 \leq i \leq k-2$. We denote by $M^{\prime}=M \cdot T$, the matching obtained by applying $T$ to $M$. Thus, for $a_{i} \in \mathcal{A}_{T}, M^{\prime}\left(a_{i}\right)=p_{i+1}$, and for $a \notin \mathcal{A}_{T}, M^{\prime}(a)=M(a)$. The matching $M \cdot C$, obtained by applying a switching cycle $C$ to $M$ is defined analogously. We also refer to $M \cdot C$ or $M \cdot T$ as a switching operation.

Figure 1 illustrates an example instance along with its switching graph.

$$
\begin{array}{llll}
a_{1}: & p_{1} & p_{2} & p_{3} \\
a_{2}: & p_{1} & p_{2} & p_{4} \\
a_{3}: & p_{1} & & \\
a_{4}: & p_{5} & p_{6} & p_{7} \\
a_{5}: & p_{5} & p_{6} & p_{7} \\
a_{6}: & p_{5} & p_{6} & p_{7}
\end{array}
$$

(a)

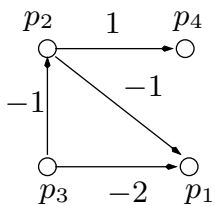

(b)

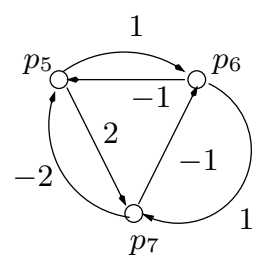

Fig. 1. (a) Preference lists of agents $\left\{a_{1}, \ldots, a_{6}\right\}$ in increasing order of ranks. (b) Switching graph $G_{M}$ with respect to rank-maximal matching $M=$ $\left\{\left(a_{1}, p_{3}\right),\left(a_{2}, p_{2}\right),\left(a_{3}, p_{1}\right),\left(a_{4}, p_{7}\right),\left(a_{5}, p_{5}\right),\left(a_{6}, p_{6}\right)\right\}$. The vertex $p_{4}$ is the only sinkvertex and the path $\left(p_{3}, p_{2}, p_{4}\right)$ is a switching path. Note that every directed cycle is a switching cycle. 


\subsection{Properties of the switching graph}

In this section, we prove several useful properties of the switching graph by characterizing switching paths and switching cycles.

In the following lemma, we show that a switching operation on a rankmaximal matching $M$ results in another rank-maximal matching in $G$.

Lemma 2. Let $T$ (resp. $C$ ) be a switching path (resp. switching cycle) in $G_{M}$. Then, $M^{\prime}=M \cdot T$ (resp. $M^{\prime}=M \cdot C$ ) is a rank-maximal matching in $G$.

Proof. We prove the lemma for a switching path $T$. A similar argument follows for a switching cycle. To show that $M^{\prime}$ is rank-maximal, we show that $M$ and $M^{\prime}$ have the same signature.

Let $T=\left\langle p_{0}, p_{1}, \ldots, p_{k-1}\right\rangle$ be a switching path in $G_{M}$. Let $\mathcal{A}_{T}=\{a \mid M(a) \in$ $T\}$. By the definition of a switch, we know that $|M|=\left|M^{\prime}\right|$ and for each $a \notin \mathcal{A}_{T}$, we have $M^{\prime}(a)=M(a)$. Thus, it suffices to show that the signatures of $M$ and $M^{\prime}$ restricted to the applicants in $\mathcal{A}_{T}$ are the same. We denote them by $\rho_{T}(M)=\left(x_{1}, x_{2}, \ldots, x_{r}\right)$ and $\rho_{T}\left(M^{\prime}\right)=\left(y_{1}, y_{2}, \ldots, y_{r}\right)$ respectively. Note that an edge of rank $i$ in $M$ contributes $-i$ to the weight of $T$, whereas one in $M^{\prime}$ contributes $i$. Further, since $T$ is a switching path, $w(T)=0$. Thus,

$$
w(T)=\left(y_{1}-x_{1}\right)+2\left(y_{2}-x_{2}\right)+\ldots+r\left(y_{r}-x_{r}\right)=0
$$

Since we consider only applicants in $\mathcal{A}_{T}$, we know that, $\sum_{i=1}^{r} x_{i}=\sum_{i=1}^{r} y_{i}$, i.e.,

$$
\sum_{i=1}^{r}\left(x_{i}-y_{i}\right)=0
$$

For contradiction, assume that $\rho_{T}(M) \succ \rho_{T}\left(M^{\prime}\right)$. That is, there exists an index $j$ such that $x_{j}>y_{j}$ and, for $1 \leq i<j$, we have $x_{i}=y_{i}$. Then, for Eqn. 2 to be satisfied, there exists an index $\ell>j$ such that $x_{\ell}<y_{\ell}$. In fact we will show the following stronger claim:

Claim. There exists an index $\ell>j$ such that $\sum_{i=1}^{\ell}\left(x_{i}-y_{i}\right)<0$.

Before proving the claim, we show how it suffices to complete the proof of the lemma. Assuming the claim, consider the reduced graph $G_{\ell}^{\prime}$ constructed in the $\ell$ th iteration of Irving et al.'s algorithm.

As $\sum_{i=1}^{\ell}\left(x_{i}-y_{i}\right)<0$, we have $\sum_{i=1}^{\ell} x_{i}<\sum_{i=1}^{\ell} y_{i}$. Thus $\left|M \cap G_{\ell}^{\prime}\right|<\left|M^{\prime} \cap G_{\ell}^{\prime}\right|$. However, by Invariant (I2) (ref. Section 2), this contradicts the fact that every rank-maximal matching restricted to any rank $\ell$ is also a maximum matching in the reduced graph $G_{\ell}^{\prime}$. This completes the proof of the lemma. We prove the claim below.

Proof (of claim): Assume the contrary, i.e. $\sum_{i=1}^{k}\left(x_{i}-y_{i}\right) \geq 0$ for all $k$. Note that this is trivially true for $k \leq j$, by our choice of $j$. Equivalently, $\sum_{i=k+1}^{r}\left(x_{i}-\right.$ $\left.y_{i}\right) \leq 0$ for all $k$. Define $T_{k}=\sum_{i=k}^{r}\left(x_{i}-y_{i}\right)$ for $1 \leq k \leq r$. Thus, to prove the 
claim, it suffices to show that there exists an index $\ell$ such that $T_{\ell}>0$. Now consider Eqn. 1, It can be rewritten as follows:

$$
\left(x_{1}-y_{1}\right)+2\left(x_{2}-y_{2}\right)+\ldots+r\left(x_{r}-y_{r}\right)=T_{1}+T_{2}+\ldots+T_{r}=0
$$

We know that $T_{1}=0$, because it is the left-side of Eqn. 2. Now, consider the term $T_{r}=x_{r}-y_{r}$. If $T_{r}=0$, we can eliminate $x_{r}$ and $y_{r}$ and get equations in $r-1$ variables. If $T_{r}>0$, then Eqn. 2 implies that the claim holds for $k=r-1$. So, without loss of generality, we can assume $T_{r}<0$. But then, to satisfy Eqn. 3, there exists an index $i, 1<i<r$, such that $T_{i}>0$. This implies that the claim holds for $\ell=i-1$. This completes the proof of the claim.

Now we address the question of recognition of switching paths and switching cycles in $G_{M}$. In Lemma 3, we show that every cycle in $G_{M}$ is in fact a switching cycle, that is, a zero-weight cycle. In Lemma 4, we characterize switching paths.

Lemma 3. Let $M$ be a $\mathrm{rmm}$ in $G$, and $C$ be a cycle in $G_{M}$. Then $w(C)=0$.

Proof. (Sketch) Let $C^{\prime}$ be the alternating cycle in $G^{\prime}$, corresponding to the cycle $C$ in $G_{M}$. To prove the Lemma, it suffices to show that, $C^{\prime}$ has an equal number of matched and unmatched edges of any rank $i$, and hence $w(C)=0$. We prove this by induction on $i$. See Appendix $\mathrm{A}$ for details.

Lemma 4. Let $M$ be a rmm in $G$, and $G_{M}$ be the switching graph with respect to $M$. Recall that $\mathcal{E}_{i}$ is the set of even vertices in the graph $G_{i}^{\prime}$ constructed in the ith iteration of Irving et al.'s algorithm. The following properties hold:

1. Let $p$ be an unmatched post in $M$. Then $p \in \mathcal{E}_{1} \cap \ldots \cap \mathcal{E}_{r+1}$ and therefore is a sink in $G_{M}$.

2. A post $p$ belongs to a sink component iff $p \in \mathcal{E}_{r+1}$. A post $p$ belongs to a non-sink component iff $p \in \mathcal{U}_{r+1}$.

3. Let $T$ be a path from a post $p$ to some sink $q$ in $G_{M}$. Then $w(T)=0$ iff $p \in \mathcal{E}_{1} \cap \ldots \cap \mathcal{E}_{r+1}$.

The proof appears in Appendix $\mathrm{A}$ In the following theorem, we prove that every rank-maximal matching can be obtained from $M$ by applying suitable switches. We include the proof in Appendix A

Theorem 1. Every rank-maximal matching $M^{\prime}$ in $G$ can be obtained from $M$ by applying to $M$ vertex-disjoint switching paths and switching cycles in $G_{M}$.

\subsection{Generating all rank-maximal pairs}

In this section we give an efficient algorithm to compute the set of rank-maximal pairs, defined below:

Definition 5. An edge $(a, p)$ is a rank-maximal pair if there exists a rankmaximal matching $M$ in $G$ such that $M(a)=p$. 
We refer to rank-maximal pairs as rmm-pairs. We show that the set of rmm-pairs can be computed in time linear in the size of the switching graph $G_{M}$ constructed with respect to any rank-maximal matching $M$. We prove the following theorem:

Theorem 2. The set of rmm-pairs for an instance $G=(\mathcal{A} \cup \mathcal{P}, E)$ can be computed in $O(\min (n+r, r \sqrt{n}) m)$ time.

Proof. We note that, by Theorem 1, an edge $(a, p)$ is a rmm-pair iff (i) $(a, p) \in M$ or, (ii) the edge $(M(a), p)$ belongs to a switching cycle in $G_{M}$ or, (iii) the edge $(M(a), p)$ belongs to a switching path in $G_{M}$.

Condition (i) can be checked by computing a rank-maximal matching $M$ which takes $O(\min (n+r, r \sqrt{n}) m)$ time. Condition (ii) can be checked by computing strongly connected components of $G_{M}$, which takes time linear in the size of $G_{M}$.

To check Condition (iii), note that a post $p$ has a zero-weight path to a sink if and only if $p \in \mathcal{E}_{1} \cap \ldots \cap \mathcal{E}_{r+1}$ by Lemma 4 (3). Moreover, all the paths from such a post $p$ to a sink have weight zero. Therefore, performing a DFS from each $p \in \mathcal{E}_{1} \cap \ldots \cap \mathcal{E}_{r+1}$ and marking all the edges encountered in the DFS (not just the tree edges) gives all the pairs which satisfy Condition (iii).

\section{Counting Rank-Maximal Matchings}

We prove that the problem of counting the number of rank-maximal matchings in an instance is \#P-complete, and give an FPRAS for the same.

\subsection{Hardness of Counting}

We prove \#P-hardness by reducing the problem of counting the number of matchings in 3-regular bipartite graphs to counting the number of rank-maximal matchings. The former was shown to be \#P-complete by Dagum and Luby 3 .

Reduction for lists with ties: First let us consider the case when preference lists may contain ties 4 . Let $H=(X \cup Y, E)$ be a 3-regular bipartite graph. We construct an instance $G$ of the rank-maximal matchings problem by setting $G=H$ and assigning rank 1 to all the edges in $E$. It is well-known that a $k$ regular bipartite graph admits a perfect matching for any $k$. It is easy to see that every perfect matching in $H$ is a rank-maximal matching in $G$ and vice versa. This proves the \#P-hardness of the problem for the case of ties.

Reduction for strict lists: Let $H=(X \cup Y, E)$ be a 3-regular bipartite graph, with $|X|=|Y|=n$. The corresponding instance $G=\left(\mathcal{A} \cup \mathcal{P}, E_{G}\right)$ of the rank-maximal matchings problem is as follows:

$\mathcal{A}=\left\{a_{x}: x \in X\right\} \cup\left\{a d_{1}, a d_{2}, \ldots, a d_{n-3}\right\} ; \mathcal{P}=\left\{p_{y}: y \in Y\right\} \cup\left\{p d_{1}, p d_{2}, \ldots, p d_{n-3}\right\}$

Here $a d_{i}, p d_{i}, 1 \leq i \leq n-3$ are dummy agents and dummy posts respectively.

To construct the preference lists of agents in $\mathcal{A}$, we fix an arbitrary ordering on the vertices in $Y$ i.e. order $: Y \rightarrow\{1, \ldots, n\}$. This assigns an ordering on the posts in $\mathcal{P}$. The preference lists of the agents can be described as below:

4 Recall that preference lists are said to contain ties if an applicant ranks two or more posts at the same rank. 
- A dummy agent $a d_{i}$ has a preference list of length one, with dummy post $p d_{i}$ as his rank 1 post.

- The preference list of an agent $a_{x}$ consists of posts $p_{y_{1}}, p_{y_{2}}, p_{y_{3}}$ ranked at $\operatorname{order}\left(y_{1}\right), \operatorname{order}\left(y_{2}\right)$, and $\operatorname{order}\left(y_{3}\right)$ respectively, where $y_{1}, y_{2}, y_{3}$ denote the 3 neighbors of $x$ in $H$. The remaining places in the preference list of $a_{x}$ are filled using the $n-3$ dummy posts.

Following Lemma (proof in Appendix $\mathbb{B}$ ) shows the correctness of the reduction.

Lemma 5. Let $H$ be a 3-regular bipartite graph and let $G$ be the rank-maximal matchings instance constructed from $H$ as above. There is a one-to-one correspondence between perfect matchings in $H$ and rank-maximal matchings in $G$.

Using Lemma 5 and our observation for ties, we conclude the following:

Theorem 3. The problem of counting the number of rank-maximal matchings in an instance is \#P-Complete for both strict and tied preference lists.

\subsection{An FPRAS for Counting Rank-Maximal Matchings}

Given the hardness result in Section 4.1, it is unlikely to be able to count the number of rank-maximal matchings in an instance in polynomial time. We now show that there exists a fully polynomial-time randomized approximation scheme (FPRAS) for the problem. We use the following result by Jerrum et al. 8]:

Theorem 4 ([8]). There exists an FPRAS for the problem of counting the number of perfect matchings in a bipartite graph.

We give a polynomial-time reduction from the problem of counting the number of rank-maximal matchings (denoted as \#RMM) to the problem of counting the number of perfect matchings in a bipartite graph (denoted as \#BPM).

Reduction from \#RMM to \#BPM: Given an instance $G=(\mathcal{A} \cup \mathcal{P}, E)$ of the rank-maximal matchings problem, we first construct another instance $H$ of the rank-maximal matchings problem, which is used to get an instance $I$ of the bipartite perfect matchings problem. The steps of the construction are as follows:

1. For every $a \in \mathcal{A}$, introduce a dummy last-resort post $\ell(a)$ ranked $r+1$. This ensures that every rank-maximal matching is $\mathcal{A}$-complete.

2. Let $M$ be any rank-maximal matching in $G$, let $G^{\prime}$ be the reduced graph obtained by Irving et al.'s algorithm (ref. Section [2).

3 . Let $k$ be the number of unmatched posts in $G^{\prime}$. Introduce $k$ dummy applicants $a d_{1}, \ldots, a d_{k}$. The preference list of each dummy applicant consists of all the posts in $G^{\prime}$ which are in $\mathcal{E}_{1} \cap \ldots \cap \mathcal{E}_{r+1}$, tied at rank $r+2$.

4. The instance $H$ consists of all the applicants in $G$ and their preference lists in $G$, together with the dummy applicants and their preference lists introduced above. The set of posts in $H$ is the same as that in $G$.

5. The instance $I$ of bipartite perfect matchings problem is simply the reduced graph $H^{\prime}$, obtained by executing Irving et al.'s algorithm on $H$. 
Correctness of the reduction follows from the following lemma, the proof (in Appendix B) uses the switching graph characterization.

Lemma 6. Let $G$ be the rank-maximal matchings instance and let $H$ and $I$ be the rank-maximal matchings instance and the bipartite perfect matchings instance respectively constructed as above. Then, the following hold:

1. Corresponding to each rank-maximal matching $M$ in $G$, there are exactly $k$ ! distinct rank-maximal matchings in $H$.

2. Each rank-maximal matching in $H$ matches all the applicants and posts, and all its edges appear in I. Hence it is a perfect matching in the instance I.

3. A matching in $G$ that is not rank-maximal has no corresponding perfect matching in $I$.

The FPRAS for \#RMM involves the following steps:

1. The reduction from \#RMM instance $G$ to \#BPM instance $I$,

2. Running Jerrum et al.'s FPRAS on $I$ to get an approximate count, say $C$, of the number of perfect matchings in $I$,

3. Dividing $C$ by $k$ ! to get an approximate count of number of rank-maximal matchings in $G$.

Steps 1 and 2 clearly work in polynomial time. For step 3, note that both $C$ and $k$ are at most $n$ ! and can be represented in $O(n \log n)$ bits, which is polynomial in the size of $G$. Therefore Step 3 also works in polynomial time. This completes the FPRAS for \#RMM problem.

\section{Popularity of Rank-Maximal Matchings}

As mentioned earlier, an instance of the rank-maximal matchings problem may admit more than one rank-maximal matching. To choose one rank-maximal matching, it is natural to impose an additional optimality criterion. Such a question has been considered earlier in the context of popular matchings by [911] and also in the context of the stable marriage problem [7. The additional notion of optimality that we impose is the notion of popularity, defined below:

Definition 6 (Popular matching). A matching $M$ is more popular than matching $M^{\prime}$ (denoted by $M \succ_{p} M^{\prime}$ ) if the number of applicants that prefer $M$ to $M^{\prime}$ is more than the number of applicants that prefer $M^{\prime}$ to $M$. A matching $M$ is popular if no matching $M^{\prime}$ is more popular than $M$.

An applicant $a$ prefers matching $M$ to $M^{\prime}$ if either (i) $a$ is matched in $M$ and unmatched in $M^{\prime}$, or (ii) $a$ is matched in both and prefers the post $M(a)$ to $M^{\prime}(a)$. We consider the following question: Given an instance of the rank-maximal matchings problem, is there a rank-maximal matching that is popular in the set of all rank-maximal matchings? We refer to such a matching as a popular rank-maximal matching. There are simple instances in which there is no popular matching; further there is no popular rank-maximal matching. However, if 
a popular rank-maximal matching exists, it seems an appealing choice since it enjoys both rank-maximality and popularity. We make partial progress on this question. Using the switching graph characterization developed in Section 3 , we give a simple algorithm to determine if a given rank-maximal matching $M$ is a popular rank-maximal matching. If not, our algorithm outputs a rank-maximal matching $M^{\prime}$ which is more popular than $M$.

Outline of the algorithm: Given a graph $G=(\mathcal{A} \cup \mathcal{P}, E)$ and a rank-maximal matching $M$ in $G$, the algorithm first constructs the switching graph $G_{M}$ corresponding to $M$. Now consider the following re-weighted graph $\tilde{G}_{M}$ where positive weights of edges in $G_{M}$ are replaced by +1 weights and negative weights by -1 . Thus a -1 weight edge $\left(p_{i}, p_{j}\right)$ in $\tilde{G}_{M}$ implies that $M\left(p_{i}\right)$ prefers $p_{j}$ to $p_{i}$.

Let $T$ be a switching path in $G_{M}$, and let $\tilde{T}$ be the corresponding path in $\tilde{G}_{M}$. It is easy to see that if $w(\tilde{T})<0$ in $\tilde{G}_{M}$, then $M^{\prime}=M \cdot T$ is more popular than $M$. Same holds for a switching cycle in $G_{M}$. Therefore, $M$ is a popular rank-maximal matching, if and only if there is no negative-weight path to sink or negative-weight cycle in $\tilde{G}_{M}$.

To check this, we use shortest path computations using Bellman-Ford algorithm in a suitably modified graph. The details of the algorithm and proof of the following lemma, which establishes correctness, appear in Appendix C]

Lemma 7. A given rank-maximal matching $M$ is popular if and only if there is no negative-weight path to a sink or a negative-weight cycle in the re-weighted switching graph.

Thus we get an $O(m n)$ time algorithm for checking whether a given rankmaximal matching is a popular rank-maximal matching, where $m$ and $n$ are number of edges and vertices in the switching graph respectively.

Acknowledgment: We thank Partha Mukhopadhyay for a proof of Lemma 2 .

\section{References}

1. D. J. Abraham, K. Cechlárová, D. F. Manlove, and K. Mehlhorn. Pareto-optimality in house allocation problems. In Proceedings of 15th ISAAC, pages 3-15, 2004.

2. D. J. Abraham, R. W. Irving, T. Kavitha, and K. Mehlhorn. Popular matchings. SIAM Journal on Computing, 37(4):1030-1045, 2007.

3. P. Dagum and M. Luby. Approximating the permanent of graphs with large factors. Theor. Comput. Sci., 102(2):283-305, 1992.

4. A. Hylland and R. Zeckhauser. The efficient allocation of individuals to positions. Journal of Political Economy, 87(2):293-314, 1979.

5. R. W. Irving. Greedy matchings. Technical Report, University of Glasgow, TR-2003-136, 2003.

6. R. W. Irving, T. Kavitha, K. Mehlhorn, D. Michail, and K. E. Paluch. Rank-maximal matchings. ACM Transactions on Algorithms, 2(4):602-610, 2006.

7. R. W. Irving, P. Leather, and D. Gusfield. An efficient algorithm for the "optimal" stable marriage. Journal of the ACM, 34(3):532-543, 1987.

8. M. Jerrum, A. Sinclair, and E. Vigoda. A polynomial-time approximation algorithm for the permanent of a matrix with nonnegative entries. J. ACM, 51(4):671-697, 2004.

9. T. Kavitha and M. Nasre. Note: Optimal popular matchings. Discrete Applied Mathematics, 157(14):3181-3186, 2009.

10. T. Kavitha and C. D. Shah. Efficient algorithms for weighted rank-maximal matchings and related problems. In Proceedings of 17th ISAAC, pages 153-162, 2006.

11. E. McDermid and R. W. Irving. Popular matchings: structure and algorithms. Journal of Combinatorial Optimization, 22(3):339-358, 2011. 
12. M. Nasre. Popular Matchings: Structure and Cheating Strategies. In Proceedings of 30th STACS, pages 412-423, 2013.

13. K. E. Paluch. Capacitated rank-maximal matchings. In Proceedings of 8th CIAC, pages $324-$ 335, 2013.

14. W. R. Pulleyblank. Handbook of combinatorics (vol. 1). chapter Matchings and Extensions, pages 179-232. MIT Press, Cambridge, MA, USA, 1995.

15. Y. Yuan. Residence exchange wanted: A stable residence exchange problem. European Journal of Operational Research, 90(3):536 - 546, 1996. 


\section{A Details from Section 3}

Lemma 3 Let $M$ be a rmm in $G$ and $C$ be a cycle in $G_{M}$, then $w(C)=0$.

Proof. Let $C$ be a cycle in $G_{M}$ and let $C^{\prime}$ denote the corresponding alternating cycle in $G^{\prime}$. To prove the Lemma statement, we show that, for any rank $i, C^{\prime}$ has an equal number of matched and unmatched edges, and hence $w(C)=0$. We use induction on $i$ to prove that for any $i$, the cycle $C^{\prime}$ has equal number of matched and unmatched edges. Let us partition the edges of $C^{\prime}$ as $X_{1} \cup \ldots \cup X_{r}$, where $X_{i}$ denotes edges of rank $i$ belonging to $C^{\prime}$. Note that, for some $i, X_{i}$ may be empty. Now for any $i$, consider $Y_{i}=\cup_{j=1}^{i} X_{j}$. We show by induction on $i$, that for each $i$, any component of $Y_{i}$ is either an even length path or the cycle $C^{\prime}$ itself.

Base case: Let $\ell$ denote the first index for which $X_{\ell}$ is non-empty. Then each $j<\ell$ trivially satisfies the induction hypothesis. If $Y_{\ell}=C^{\prime}$, we are done, since $C^{\prime}$ is an alternating cycle, with equal number of unmatched and matched edges, all of rank $\ell$. If $Y_{\ell} \neq C^{\prime}$, then for contradiction, let $Y_{\ell}$ contain an odd length alternating path $T=\left\langle a_{1}, p_{1}, a_{2}, p_{2}, \ldots, a_{k}, p_{k}\right\rangle$. Since all the other edges in $C^{\prime}$ are of rank greater than $\ell$, and they are incident on $a_{1}$ and $p_{k}$, both $a_{1}$ and $p_{k}$ must belong to $\mathcal{E}_{\ell}$ in $G_{\ell}^{\prime}$ at the end of the $\ell$ th iteration of Irving et al.'s algorithm. However, since $T$ is present in $G^{\prime}$ it must be present in $G_{\ell}^{\prime}$. Note that both $a_{1}$ and $p_{k}$ belong to $\mathcal{E}_{\ell}$ and the path $T$ is an alternating path of odd length. Now consider labeling the vertices of $T$ as $\mathcal{O}_{\ell}$ or $\mathcal{E}_{\ell}$ from both $a_{1}$ and $p_{k}$. It is clear that we either encounter an $\mathcal{O}_{\ell} \mathcal{O}_{\ell}$ edge which must have been deleted in Irving et al.'s algorithm or an $\mathcal{E}_{\ell} \mathcal{E}_{\ell}$ edge which cannot be present in $G$ (by Lemma 1(c)). Thus in either case, we get a contradiction. Hence $T$ must be an even length path, with equal number of matched and unmatched rank $\ell$ edges.

Induction step: Assuming the induction hypothesis for some $\ell<r$, the proof for $Y_{\ell+1}$ is similar to that of base case.

The above implies that, for every $X_{i}, C^{\prime}$ has $\left|X_{i}\right| / 2$ matched and unmatched edges. Hence the corresponding cycle $C$ in $G_{M}$ has zero weight, and $M \cdot C$ is a rank-maximal matching.

Lemma 4 Let $M$ be a $\mathrm{rmm}$ in $G$ and $G_{M}$ be the switching graph with respect to $M$. The following properties hold:

1. Let $p$ be an unmatched post in $M$. Then $p \in \mathcal{E}_{1} \cap \ldots \cap \mathcal{E}_{r+1}$ and therefore is a sink in $G_{M}$.

2. A post $p$ belongs to a sink component iff $p \in \mathcal{E}_{r+1}$. A post $p$ belongs to a non-sink component iff $p \in \mathcal{U}_{r+1}$.

3. Let $T$ be a path from a post $p$ to some sink $q$ in $G_{M}$. Then $w(T)=0$ iff $p \in \mathcal{E}_{1} \cap \ldots \cap \mathcal{E}_{r+1}$. 
Proof. 1. The proof follows by observing that every rank-maximal matching keeps vertices in $\mathcal{O}_{i} \cup \mathcal{U}_{i}$ matched for every $i=1 \ldots r+1$. Thus if $p$ is unmatched in a rank-maximal matching $M$, then $p \in \mathcal{E}_{1} \cup \ldots \cup \mathcal{E}_{r+1}$.

2. Consider a post $p \in \mathcal{E}_{r+1}$. If $p$ is unmatched in $M$, then by 1 above, $p$ is a sink vertex and therefore belongs to a sink component. Now, assume that $p$ is matched but since it belongs to $\mathcal{E}_{r+1}, p$ has an even length alternating path starting at an unmatched node $p^{\prime}$ with respect to $M$ in $G^{\prime}$. Let the alternating path be denoted by $\left\langle p=p_{1}, a_{1}, \ldots, p_{k}, a_{k}, p_{k+1}=p^{\prime}\right\rangle$. Note that for every $i=1, \ldots k$, we have $M\left(a_{i}\right)=p_{i}$. Further, every unmatched edge $\left(a_{i}, p_{i+1}\right)$ is of the form $\mathcal{O}_{r+1} \mathcal{E}_{r+1}$. Therefore no such unmatched edge gets deleted in the $(r+1)$ st iteration of Irving et al.'s algorithm. This implies that the directed path $\left\langle p=p_{1}, p_{2}, \ldots, p_{k+1}=p^{\prime}\right\rangle$ is present in $G_{M}$. Thus, $p$ belongs to the sink component that contains $p^{\prime}$.

To prove the other direction let $\mathcal{X}$ be a sink component in $G_{M}$ and $p^{\prime}$ be a sink in $\mathcal{X}$. For the sake of contradiction, let $p^{\prime} \in \mathcal{X}$ and $p \in \mathcal{U}_{r+1}$. Recall that $\mathcal{O}_{r+1} \cap \mathcal{P}=\emptyset$.

Now since $p$ and $p^{\prime}$ lie in the same component, there is an (undirected) path between $p$ and $p^{\prime}$ in the underlying undirected component of $\mathcal{X}$. Let $\left\langle p=p_{1}, p_{2}, \ldots, p_{k}=p^{\prime}\right\rangle$ denote this undirected path. Since $p_{1} \in \mathcal{U}_{r+1}$ and $p_{k} \in \mathcal{E}_{r+1}$, it implies that there exists an index $i, 1 \leq i \leq k-1$, such that $p_{i} \in \mathcal{U}_{r+1}$ and $p_{i+1} \in \mathcal{E}_{r+1}$. Note that, by the above argument, $p_{i+1}$ has a directed path $T$ to some sink $q$ in $\mathcal{X}$.

Consider the two possible directions for the edge between $p_{i}$ and $p_{i+1}$ in $G_{M}$ :

(a) If the edge is directed from $p_{i}$ to $p_{i+1}$ in $G_{M}$, then the path $T$ from $p_{i+1}$ to the sink $q$ in $\mathcal{X}$ can be prefixed with the edge $\left(p_{i}, p_{i+1}\right)$ to get a directed path from $p_{i}$ to $q$. This implies that there is an even-length alternating path with respect to $M$ in $G^{\prime}$ from $q$ to $p_{i}$. This contradicts the fact that $p_{i} \in \mathcal{U}_{r+1}$.

(b) Finally, if the edge is directed from $p_{i+1}$ to $p_{i}$ in $G_{M}$, then it implies that $p_{i+1}$ is matched in $M$ and let $M\left(p_{i+1}\right)=a_{i+1}$. Since $p_{i+1} \in \mathcal{E}_{r+1}$ this implies that $a_{i+1} \in \mathcal{O}_{r+1}$. Thus the edge $\left(p_{i+1}, p_{i}\right)$ in $G_{M}$ implies that there is an $\mathcal{O}_{r+1} \mathcal{U}_{r+1}$ edge $\left(a_{i+1}, p_{i}\right)$ in the graph $G^{\prime}$. However, such an $\mathcal{O}_{r+1} \mathcal{U}_{r+1}$ edge should have been deleted by Irving et al.'s algorithm Hence such an edge cannot be present in $G_{M}$ contradicting the fact that $p \in \mathcal{U}_{r+1}$. Thus, every post $p$ belonging to a sink component belongs to $\mathcal{E}_{r+1}$.

The above proof along with the fact that $\mathcal{P} \cap \mathcal{O}_{r+1}=\emptyset$ immediately implies that a post $p$ belongs to a non-sink component iff $p \in \mathcal{U}_{r+1}$.

3. First assume that $p$ has a path $T$ to a sink and $w(T)=0$. Our goal is to show that $p \in \mathcal{E}_{1} \cap \ldots \cap \mathcal{E}_{r+1}$. Since $p$ has a path to a sink, $p \in \mathcal{E}_{r+1}$. Assume for the sake of contradiction that $p \in \mathcal{O}_{i} \cup \mathcal{U}_{i}$ for some $i \leq r$. Let $M^{\prime}=M \cdot T$ be the matching obtained by switching along the path $T$. Since $T$ has zero weight, from Lemma 2, we know that the matching $M^{\prime}$ is a rank-maximal matching in $G$. Note that, since $q$ is unmatched in $M, p$ is unmatched in $M^{\prime}$. Thus we have obtained a rank-maximal matching $M^{\prime}$ in $G$ which leaves $p$ unmatched. By the invariants of Irving et al.'s algorithm, 
mentioned in Section 2, we know that every vertex belonging to $\mathcal{O}_{i} \cup \mathcal{U}_{i}$ remains matched in every rank-maximal matching of $G$. However, we have already obtained a matching, namely $M^{\prime}$, which leaves $p$ unmatched. This contradicts the assumption that $p \in \mathcal{O}_{i} \cup \mathcal{U}_{i}$ for some $i$, and completes the proof that $p \in \mathcal{E}_{1} \cap \ldots \cap \mathcal{E}_{r+1}$.

Finally, consider the other direction, that is assume that $p \in \mathcal{E}_{1} \cap \ldots \cap \mathcal{E}_{r+1}$ and $p$ has a path $T$ to a sink. To show that $w(T)=0$, we use arguments similar to proof of Lemma 3

This completes the proof of the Lemma.

Theorem 1 Every rank-maximal matching $M^{\prime}$ in $G$ can be obtained from $M$ by applying to $M$ vertex-disjoint switching paths and switching cycles in $G_{M}$.

Proof. Consider any rank-maximal matching $M^{\prime}$ in $G$. We show that $M^{\prime}$ can be obtained from $M$ by applying a set of vertex-disjoint switching paths and switching cycles of $G_{M}$. Consider $M \oplus M^{\prime}$ which is a collection of vertex-disjoint paths and cycles in $G$. Also note that the cycles and paths contain alternating edges of $M$ and $M^{\prime}$. We show that the paths and cycles in $M \oplus M^{\prime}$ are switching paths and switching cycles in $G_{M}$.

From the invariants of Irving et al.'s algorithm mentioned in Section 2, all the edges of $M$ and $M^{\prime}$ are also present in $G^{\prime}$. A cycle in $M \oplus M^{\prime}$ has alternating edges of $M$ and $M^{\prime}$, and hence has a corresponding directed cycle in $G_{M}$. As proved in Lemma 3, every cycle in $G_{M}$ is a switching cycle.

Now we consider paths in $M \oplus M^{\prime}$. All the paths are of even length, since all the rank-maximal matchings are of the same cardinality. Let $T_{G}=$ $\left\langle p_{1}, a_{1}, \ldots, p_{k}, a_{k}, p_{k+1}\right\rangle$ be any even-length path in $M \oplus M^{\prime}$ with $p_{k+1}$ unmatched in $M$ and $p_{1}$ unmatched in $M^{\prime}$. For every $1 \leq i \leq k$, let $M\left(p_{i}\right)=a_{i}$. It is easy to see that the path $T=\left\langle p=p_{1}, p_{2}, \ldots, p_{k+1}=p^{\prime}\right\rangle$ is present in $G_{M}$ and it ends in a sink $p^{\prime}$. Our goal is to show that $w(T)=0$. For this, we prove that $p_{1} \in \mathcal{E}_{1} \cap \ldots \cap \mathcal{E}_{r+1}$. Note that $M^{\prime}$ is a rank-maximal matching in $G$ and $M^{\prime}$ leaves the post $p=p_{1}$ unmatched. As every post in $\mathcal{O}_{i} \cup \mathcal{U}_{i}$ for any $i$ is matched in every rank-maximal matching, $p_{1} \notin \mathcal{O}_{i} \cup \mathcal{U}_{i}$ for $1 \leq i \leq r+1$. Therefore $p_{1} \in \mathcal{E}_{1} \cap \ldots \cap \mathcal{E}_{r+1}$; Thus, using Lemma 4, we can conclude that the path $T$ has weight $w(T)=0$ in $G_{M}$, and hence is a switching path in $G_{M}$.

Applying these switching paths and cycles to $M$ gives us the desired matching $M^{\prime}$, thus completing the proof.

\section{B Proofs from Section 4}

Lemma 5 Let $H$ be a 3-regular bipartite graph and let $G$ be the corresponding rank-maximal matchings instance constructed by the reduction in Section 4 . A matching $M$ is a perfect in $H$ iff $M$ is a rank-maximal matching in $G$. 
Proof. A perfect matching $M$ in $H$ can be extended in a unique way to a perfect matching $M^{\prime}$ in $G$ as follows: $M^{\prime}=M \cup\left\{\left(a d_{i}, p d_{i}\right) \mid 1 \leq i \leq n-3\right\}$. The matching $M^{\prime}$ has the following $n$-tuple as its signature: $\sigma\left(M^{\prime}\right)=(n-2,1,1, \ldots, 1)$. Hence, a rank-maximal matching in $G$ should have a signature that is at least as good as $\sigma\left(M^{\prime}\right)$. We argue that $\sigma\left(M^{\prime}\right)$ is the best possible signature in $G$.

Consider the posts in $\mathcal{P}$ that are rank 1 posts for some applicant $a \in \mathcal{A}$. There are exactly $n-2$ such posts: the $n-3$ dummy posts $p d_{1}, \ldots, p d_{n-3}$ and one post $p_{y}$ such that $\operatorname{order}\left(p_{y}\right)=1$. Therefore, any rank-maximal matching in $G$ cannot match more than $n-2$ applicants to their rank-1 posts. Moreover, all these $n-3$ posts are odd or unreachable in the graph on rank 1 edges and hence are always matched to applicants that treat them as their rank 1 posts. At each of the ranks $2 \leq i \leq n$, there is exactly one post $p_{y} \in \mathcal{P}$ that is ranked $i$. Thus, it is easy to see that $\sigma\left(M^{\prime}\right)$ is the best possible signature for any matching in $G$. Therefore $M^{\prime}$ is a rank-maximal matching in $G$. Further, note that $M^{\prime}$ was obtained by extending a perfect matching $M$ in $H$. This also implies that, for every perfect matching in $H$, there is a unique rank-maximal matching in $G$.

Now consider a rank-maximal matching $M$ in $G$. We claim that such a matching has to include the edges $\left\{\left(a d_{i}, p d_{i}\right) \mid 1 \leq i \leq n-3\right\}$. If not, then for some $i=1 \ldots n-3$ applicant $a d_{i}$ remains unmatched and therefore $\sigma(M) \prec(n-2,1, \ldots, 1)$. Similarly, to achieve the signature $(n-2,1, \ldots, 1)$, all applicants $a_{x}$ and therefore all posts $p_{y}$ should be matched amongst themselves. Thus, the matching $M^{\prime}=M \backslash\left\{\left(a d_{i}, p d_{i}\right) \mid 1 \leq i \leq n-3\right\}$ is a perfect matching in $H$. This shows that there is a one to one correspondence between the rankmaximal matchings in $G$ and perfect matching in $H$.

Lemma $6 \quad$ Let $G$ be the rank-maximal matchings instance and let $H$ and $I$ be the rank-maximal matchings instance and the bipartite perfect matchings instance respectively as constructed in Section 4.2. Then, the following hold:

1. Corresponding to each rank-maximal matching $M$ in $G$, there are exactly $k$ ! distinct rank-maximal matchings in $H$.

2. Each rank-maximal matching in $H$ matches all the applicants and posts, and all its edges appear in I. Hence it is a perfect matching in the instance I.

3. A matching in $G$ that is not rank-maximal has no corresponding perfect matching in $I$.

Proof. 1. It is easy to see that Irving et al.'s algorithm proceeds on $H$ exactly as on $G$ for $r+1$ iterations. Hence signature of a rank-maximal matching in $H$ is the same as that in $G$ for first $r+1$ co-ordinates. In $(r+2)$ nd iteration, the newly added applicants and their edges are considered. This iteration has a complete bipartite graph on rank $r+2$ edges, with $k$ applicants on left and $\left|\mathcal{E}_{1} \cap \ldots \cap \mathcal{E}_{r+1}\right|$ posts on right, with exactly $k$ posts unmatched. So there are $k$ ! ways of matching these newly added applicants amongst the unmatched posts. Thus, corresponding to each rank-maximal matching constructed in the first $r+1$ iterations (and hence in $G$ ), there are $k$ ! rankmaximal matchings in $G_{r+2}$. 
2. This is immediate from the construction.

3. We show that every perfect matching in $I$ is a rank-maximal matching in $H$. Let there be a perfect matching $M$ in $I$ which is not rank-maximal in $H$. Consider a rank-maximal matching $N$ in $H$. As $M$ and $N$ are both perfect matchings, $M \oplus N$ is a collection of vertex-disjoint cycles with alternate edges of $M$ and $N$. Hence these cycles are switching cycles in the switching graph $H_{N}$. But all the switching cycles in $H_{N}$ have weight 0. By Lemma 2, this contradicts the assumption that $M$ is not rank-maximal.

This completes the proof of the lemma.

\section{Details from Section 5}

\section{The algorithm for popular rank-maximal matching}

1. Make $M \mathcal{A}$-complete by adding dummy last-resort posts at rank $r+1$ as described in Section 2. Here $r$ is the maximum length of any preference list in $G$. Remove those edges $\left(p_{i}, p_{j}\right)$ from $G_{M}$, where $\left(M\left(p_{i}\right), p_{j}\right)$ is not a rank-maximal pair.

2. Construct a re-weighted graph $\tilde{G}_{M}$ by replacing every positive-weight in $G_{M}$ by +1 and negative weight by -1 .

3. For each sink-component $\mathcal{X}_{i}$ in the $\tilde{G}_{M}$, add a source vertex $s_{i}$ and a sink $t_{i}$. For every non-sink vertex $p \in \mathcal{E}_{1} \cap \ldots \cap \mathcal{E}_{r+1}$ add an edge $\left(s_{i}, p\right)$ of weight zero. For every sink $p$, add an edge $\left(p, t_{i}\right)$ of weight zero. Thus $s_{i}$ and $t_{i}$ are new source and sink for the component $\mathcal{X}_{i}$. For each non-sink component of $\tilde{G}_{M}$, choose an arbitrary vertex $v$ as source.

4. Run Bellman-Ford algorithm from each source to find if there is a negativeweight cycle in any component or a negative-weight $s_{i}$ to $t_{i}$ path in any sink-component $\mathcal{X}_{i}$.

5. If there exists a negative-weight path $T$ or cycle $C$ in the above graph, then conclude that $M$ is not a popular rank-maximal matching. Find such a cycle $C$ or path $T$ and output $M^{\prime}=M \cdot C$ or $M^{\prime}=M \cdot T$ respectively, as a rank-maximal matching more popular than $M$. Otherwise $M$ is a popular rank-maximal matching.

Lemma 7 A given rank-maximal matching $M$ is popular if and only if there is no negative weight path to a sink or a negative-weight cycle in the re-weighted switching graph.

Proof. We prove that, if there is a negative-weight cycle or a negative-weight path to a sink in $\tilde{G}_{M}$, then a matching $N$ obtained by switching along such a path or cycle is more popular than $M$.

Consider a negative-weight cycle, say $C=\left(p_{1}, \ldots, p_{k}, p_{1}\right)$ in $\tilde{G}_{M}$. Then All the applicants, such that weight of $\left(M\left(p_{i}\right), p_{i+1}\right)$ is negative (positive), get a post of strictly better (worse) rank in $M \cdot C$. As $C$ has more edges of negative-weight than those of positive-weight, more applicants prefer $M \cdot C$ over $M$ than those 
who prefer $M$ over $M \cdot C$. Thus $M \cdot C$ is more popular than $M$ and hence $M$ is not popular. Similar argument holds for a path to sink.

Now, let there be a rank-maximal matching $N$ that is more popular than $M$. The matching $N$ can be obtained from $M$ by application of a set $S$ of vertex-disjoint switching cycles/paths. Those applicants $a$ such that $M(a)$ is not a part of any switching cycle or path are indifferent between $M$ and $N$. An applicant $a$ prefers $N(M)$ over $M(N)$ when he gets a better (worse) ranked post in $N(M)$ than in $M(N)$. But $a$ gets $M(a)$ in $M$ and a post $p$ in $N$, where $(M(a), p)$ is a part of a switching cycle/path in $S$. But then this edge should have a negative (positive) weight in $G_{M}$ and hence a $-1(+1)$ weight in $\tilde{G}_{M}$. As more applicants prefer $N$ over $M$, there should be more negative-weight edges than positive-weight edges in the switching paths/cycles in $S$. Hence there is at least one switching cycle/path in $S$ that has more negative- weight edges than positive-weight edges, which is a negative-weight cycle/path in $\tilde{G}_{M}$.

Note that removal of those edges from $G_{M}$ which do not correspond to rankmaxi mal pairs ensures that a negative-weight cycle, if present, is reachable from one of the chosen sources. 